DOI: 10.12731/2070-7568-2020-3-52-66

УДК 378

\title{
ГРУППИРОВКА ФАКТОРОВ, ВЛИЯЮЩИХ \\ НА ИЗМЕНЕНИЕ ЭКОНОМИЧЕСКИХ ОТНОШЕНИЙ \\ КРЕДИТНЫХ ОРГАНИЗАЦИЙ И БИЗНЕСА \\ В УСЛОВИЯХ ЦИФРОВОЙ ЭКОНОМИКИ
}

\section{Генеральницкая Е.И.}

Состояние вопроса: Экономическая наука насчитывает большое число трудов, посвященных изучению факторов, оказывающих влияние на кредитные организации и предпринимательские структуpы. Однако в условиях иифровой экономики назрела необходимость по уточнению теоретической базы, учитывающей ее особенности. Помимо этого, необходимо разработать группировку факторов, оказывающих влияние на изменение экономических отношений кредитных организациии и предпринимательских структур.

Методы исследования: системные, структурные и сравнительные методы анализа, методы научной абстракиии, метод группировки.

Результаты исследования: в рамках данной статьи сделана группировка факторов, оказывающих влияние на экономические отношения предпринимательских структур и кредитных организаций в условиях ичифровой экономики. Внешние факторы поделены на объективные и субъективные. Внутренние факторы - на совместные и специфические.

Обсуждение: предложенная группировка учитывает как спеиифику экономических отношений, так и цифровую реальность при этом во многом опирается на существующую теоретическую базу. Факторы представлены в качестве многоуровневого списка для более глубокой детализации группировки. Заключение: группировка внутренних и внешних факторов, обусловивших изменение экономических отнотений кредитных организаций и предпринимательских 
структур в условиях циифровой экономики может быть востребована при поиске решений для повышения цуифровизации экономики Российской Федерации. Поскольку цฺифровизаџия банковского сектора протекает более быстрыми темпами, чем предпринимательских структур, возможно построение инклюзивной модели, в которой банковский сектор взял бы на себя ведущую роль интегращии предпринимательских структур в ичифровую среду.

Ключевые слова: группировка факторов; изменение экономических отношений; кредитные организации; предпринимательские структуры; ичифровая экономика.

\section{GROUPING OF FACTORS THAT CAUSED THE CHANGE IN ECONOMIC RELATIONS BETWEEN CREDIT ORGANIZATIONS AND BUSINESS IN CONTEXT OF DIGITAL ECONOMY}

\section{Generalnitskaia E.I.}

State of the issue: Economic science has a large number of works devoted to the study of factors that influence credit institutions and business structures. In the conditions of the digital economy, there is a need to clarify the theoretical base, taking into account new economy's features. In addition, it is necessary to work out the grouping of factors that influence the change in the economic relations of credit institutions and business structures.

Research methods: systemic, structural and comparative methods of analysis, methods of scientific abstraction, grouping method.

Results of the study: Within the framework of this article, a grouping of factors influencing the economic relations of entrepreneurial structures and credit institutions in the digital economy was made. External factors are divided into objective and subjective. Internal factors - into joint and specific.

Discussion: proposed grouping takes into account both the specifics of economic relations and digital reality, while largely based on the existing theoretical base. The factors are presented as a multilevel list for more detailed grouping. 
Conclusion: grouping of internal and external factors that caused changes in economic relations of credit institutions and business structures in the digital economy may be used for finding solutions to increase the digitalization of the economy of the Russian Federation. Since the digitalization of the banking sector is proceeding at a faster pace than the digitalization of entrepreneurial structures, it is possible to build an inclusive model in which the banking sector would take the leading role of integrating entrepreneurial structures into the digital environment.

Keywords: grouping of factors; changing of economic relations; credit institutions; entrepreneurial structures; digital economy.

\section{Введение}

Экономическая наука насчитывает огромное количество трудов, затрагивающих все узкие аспекты хозяйственной деятельности. Несмотря на это, происходящая в настоящее время цифровая трансформация [5] [14] [15], диктует необходимость актуализации теоретической базы по некоторым вопросам, в числе которых экономические отношения кредитных организаций и предпринимательских структур в условиях цифровой экономики. Большинство трудов, посвященных изучению вопросов влияния различных факторов на трансформацию кредитных организаций и предпринимательских структур, не учитывают вопросов их взаимодействия, или экономических отношений, равно как и особенностей функционирования в цифровой экономике. Изучение проблематики цифровизации предполагает интеграцию предыдущих исследований в современную реальность для создания теоретической базы, учитывающей все нюансы взаимодействия кредитных организаций и предпринимательских структур в новой цифровой реальности. В данной статье на основании анализа трудов, посвященных исследованию факторов, оказывающих влияние на трансформацию кредитных организаций и предпринимательских структур, предлагается подход к группировке факторов, обусловивших изменение экономических отношений кредитных организаций и предпринимательских структур в условиях цифровой экономики. 


\section{Материалы и методы исследования}

В данной статье для достижения поставленной цели были использованы методы: группировки, сравнения, анализа, синтеза.

\section{Результаты исследования}

Для достижения поставленной цели были проанализированы следующие исследования, посвященные вопросам трансформации кредитных организаций и предпринимательских структур:

- В части факторов, оказывающих влияние на кредитные организации: В.А. Боровкова «факторы, влияющие на развитие банковской системы» [4], В.Б. Главацкий и Р.В. Зике «факторы, влияющие на развитие кредитных организаций» [6], П.Ф. Колесова «факторы, влияющие на конкурентоспособность кредитных организаций» [10], Инес Гхазоуани Бен Амеур и Сониа Моусса Мхири «факторы, оказывающие влияние на прибыльность кредитных организаций» [17].

- В части факторов, оказывающих влияние на предпринимательские структуры: И.П. Хунгуреева, Н.Э. Шабыкова, И.Ю. Унгаева «факторы, влияющие на эффективное функционирование предпринимательских структур» [16], О.А. Зингер, А.В. Ильясова «факторы, влияющие на устойчивое развитие предпринимательских структур» [8], А.В. Александров «факторы конкурентоспособности предпринимательских структур» [2], Суятха Тривикрам «факторы деловой среды, влияющие на успешность деятельности предпринимательских структур» [18].

Анализ перечисленных исследований позволил установить, что для кредитных организаций и для предпринимательских структур используются аналогичные классификации. В качестве основного признака классификации как для кредитных организаций, так и для предпринимательских структур выбран признак - по способу возникновения (во всех исследованиях факторы делятся на внутренние и внешние). Помимо признака классификации «по способу возникновения» возможно также систематизировать факторы в зависимости от направления деятельности (позитивные и негатив- 
ные), по возможности определения размера влияния (поддающиеся количественному измерению и не поддающиеся), по возможности управления, длительности действия и т.д.

Некоторые классификации имеют свои особенности, к примеру, носят не описательный характер, а вводят конкретную систему показателей, позволяющую дать оценку текущей ситуации, или авторы предлагают разделить факторы внешней среды на микро- и макроуровни.

Необходимо отметить, что авторы вышеуказанных исследований описывали классификацию факторов, во-первых, для разных субъектов (кредитных организаций и организаций предпринимательского сектора), во-вторых, рассматривали различные критерии для выделения факторов. В качестве критериев классификации факторов использовались схожие по смыслу формулировки, такие как: факторы, влияющие на развитие банковской системы; факторы, влияющие на развитие российских кредитных организаций; факторы, влияющие на устойчивое развитие промышленных предприятий; факторы, влияющие на эффективное функционирование предприятия; факторы, оказывающие влияние на прибыльность кредитных организаций; факторы, влияющие на успешность деятельности предпринимательских структур, а также факторы, влияющие на конкурентоспособность коммерческих банков и факторы конкурентоспособности предпринимательских структур.

Учитывая вышеизложенное, отметим, что все перечисленные классификации не подходят для изучения экономических отношений кредитных организаций и предпринимательских структур в условиях цифровой экономики, поскольку, во-первых, рассматривают в качестве объекта исследования только одну сторону экономических отношений, во-вторых, не исследуют специфические факторы, вызывающие изменение экономических отношений между объектами исследования именно под воздействием процессов цифровизации. Поэтому с целью изучения экономических отношений между кредитными организациями и предпринимательскими структурами в условиях цифровой экономики необходимо предложить классификацию факторов, оказывающих влияние на трансформацию экономи- 
ческих отношений кредитных организаций и предпринимательских структур в условиях цифровой экономики.

Для идентификации факторов, обусловивших изменение экономических отношений именно под воздействием цифровой экономики, уточним, что под цифровизацией экономических отношений понимаются те изменения, которые произошли вследствие конвергенции большого числа научно-технических инноваций, которые появились благодаря интернет технологиям [13].

Как отмечалось выше, обзор классификации факторов, влияющих на деятельность кредитных организаций и организаций предпринимательского сектора, позволил установить, что в качестве основного признака классификации используется признак «по способу возникновения», поэтому считаем целесообразным поделить факторы, обусловившие изменение экономических отношений кредитных организаций и организаций предпринимательского сектора в условиях цифровой экономики, на внешние и внутренние. Группировка внешних факторов, обусловивших изменение экономических отношений кредитных организаций и предпринимательских структур в условиях цифровой экономики представлена в таблице 1.

Таблицуа 1.

Группировка внешних факторов, обусловивших изменение экономических отношений кредитных организаций и предпринимательских структур в условиях цифровой экономики

\begin{tabular}{|c|c|c|}
\hline \multicolumn{3}{|l|}{ 1. Внешние } \\
\hline \multirow{7}{*}{$\begin{array}{l}\text { 1.1. Объективные } \\
\text { (не зависящие от } \\
\text { объектов исследо- } \\
\text { вания) }\end{array}$} & \multicolumn{2}{|l|}{ 1.1.1. Экономические } \\
\hline & \multicolumn{2}{|l|}{ 1.1.2. Политические } \\
\hline & \multicolumn{2}{|l|}{ 1.1.3. Правовые } \\
\hline & \multicolumn{2}{|c|}{ 1.1.4. Благоприятный инвестиционный климат } \\
\hline & \multicolumn{2}{|c|}{ 1.1.5. Уровень развития цифровой инфраструктуры } \\
\hline & \multicolumn{2}{|c|}{ 1.1.6. Научно-технический прогресс } \\
\hline & \multicolumn{2}{|c|}{ 1.1.7. Социально-психологические } \\
\hline \multirow{2}{*}{$\begin{array}{l}\text { 1.2. Субъективные } \\
\text { (зависящие от объ- } \\
\text { ектов исследования) }\end{array}$} & $\begin{array}{l}\text { 1.2.1. Для предпринима- } \\
\text { тельских структур }\end{array}$ & $\begin{array}{l}\text { 1.2.1.1. Отраслевая } \\
\text { специфика предприятия }\end{array}$ \\
\hline & $\begin{array}{l}\text { 1.2.2. Для кредитных } \\
\text { организаций }\end{array}$ & $\begin{array}{l}\text { 1.2.2.1. Интеграция в цифро- } \\
\text { вую институциональную сре- } \\
\text { ду банковского сектора }\end{array}$ \\
\hline
\end{tabular}


Анализ данных, представленных в таблице, позволяет заключить, что внешние факторы поделены на объективные и субъективные (не зависящие и зависящие от объектов исследования соответственно).

К объективным отнесены следующие факторы:

1. Экономические факторы подразумевают под собой условия, в которых функционирует предприятия и кредитные организации, характеризуются такими показателями как уровень и динамика ВВП, промышленного производства, инфляции, денежно-кредитная политика и т.д.

2. Политические факторы включают политическую стабильность, защиту прав собственности, политику государства в области информационных технологий, налоговую политику и т.д.

3. К правовым факторам относятся: законодательное регулирование предпринимательской и банковской деятельности, законодательство в области информационных технологий и т.д.

4. Позитивный инвестиционный является фактором, формируемым политико-правовой средой, но ввиду его важности выделен отдельно. Цифровизация как организаций предпринимательского сектора, так и кредитных организаций требует больших капитальных вложений. Так, к примеру, по данным отчетности МСФО, за три года такие статьи затрат кредитных организаций, как телекоммуникационные, компьютерные и ИТ-расходы, выросли в ряде крупных банков в 1,5-3 раза [11].

6. Под уровнем развития цифровой инфраструктуры подразумевается уровень распространения сетей связи, в частности сети интернет, создание системы российских центров обработки данных (ЦОД), распространение технологий, сетей, обеспечивающих сбор, обработку, хранение и передачу данных и т.д.

7. Научно-технический прогресс предполагает высокий уровень развития науки и техники, наличие современных технологий, необходимых для цифровизации экономики.

8. Под социально-психологическими факторами подразумевается социокультурное развитие, характеризующееся отсутствием сопротивления изменениям, осознанием необходимости интеграции в цифровую среду. 
Субъективные факторы предлагается представить отдельно для кредитных организаций и предпринимательских структур.

В качестве субъективного фактора для предпринимательских структур обозначена отраслевая специфика предприятия. Это объясняется тем, что уровень цифровизации предприятия тесно связан с родом его деятельности: в соответствии со статистикой некоторые отрасли являются аутсайдерами.

В качестве субъективного фактора для кредитных организаций рассматривается интеграция в цифровую институциональную среду, созданную для банковского сектора. Имеется в виду интеграция в различные цифровые платформы, использование цифровой экосистемы. К примеру, использование кредитной организацией облачной инфраструктуры, центров обработки данных.

Группировка внутренних факторов, обусловивших изменение экономических отношений кредитных организаций и предпринимательских структур в условиях цифровой экономики представлена в таблице 2 .

Таблица 2.

Группировка внутренних факторов, обусловивших изменение экономических отношений кредитных организаций и предпринимательских структур в условиях цифровой экономики

\begin{tabular}{|c|c|c|}
\hline \multicolumn{3}{|c|}{ 1. Внутренние } \\
\hline \multirow{4}{*}{ 2.1. Совместные } & \multicolumn{2}{|c|}{ 2.1.1. Уровень цифровизации } \\
\hline & \multicolumn{2}{|c|}{$\begin{array}{l}\text { 2.1.2. Политика в области информационных технологий и } \\
\text { стратегия развития }\end{array}$} \\
\hline & \multicolumn{2}{|c|}{ 2.1.3. Кадровый потенциал } \\
\hline & \multicolumn{2}{|c|}{ 2.1.4. Экономическая целесообразность цифровизации } \\
\hline \multirow{4}{*}{$\begin{array}{l}\text { 2.2. Специфиче- } \\
\text { ские }\end{array}$} & \multirow{2}{*}{\begin{tabular}{|l|}
$\begin{array}{l}\text { 2.2.1. Для пред- } \\
\text { принимательских } \\
\text { структур }\end{array}$ \\
\end{tabular}} & 2.2.1.1. Размер предприятия \\
\hline & & $\begin{array}{l}\text { 2.2.1.2. Экстраполяция взаимодействия } \\
\text { в качестве физических лиц }\end{array}$ \\
\hline & \multirow[t]{2}{*}{$\begin{array}{l}\text { 2.2.2. Для кредит- } \\
\text { ных организаций }\end{array}$} & $\begin{array}{l}\text { 2.2.2.1. Специализация } \\
\text { (изменение традиционной модели } \\
\text { банковской деятельности) }\end{array}$ \\
\hline & & 2.2.2.2. Клиентоориентированность \\
\hline
\end{tabular}

Анализ данных, представленных в таблице, позволяет заключить что внутренние факторы поделены на совместные и специфические. 
При этом под внутренними факторами подразумеваются факторы, формирующиеся участниками экономических отношений (как кредитными организациями, так и организациями предпринимательского сектора). К «специфическим факторам» относятся факторы, касающиеся только одного из участников экономических отношений (кредитных организаций или предпринимательских структур), но оказывающих существенное влияние на их экономические отношения. К «совместным факторам» - факторы, влияющие на обоих участников экономических отношений

В качестве совместных факторов выделяются:

1. Уровень цифровизации кредитной организации/организации предпринимательского сектора (анализ таких показателей, как наличие интернета, его скорость, наличие веб-сайта, использование облачных сервисов, а также направления их использования равно как и направления использования интернета, использование технологий электронного обмена данными и т.д.).

2. Политика в области информационных технологий и стратегия развития (рассмотрение вопросов цифровизации в качестве приоритетных направлений для развития).

3. Кадровый потенциал (внедрение и обслуживание цифровых технологий предполагает наличие квалифицированных кадров).

4. Экономическая целесообразность цифровизации (возможность снижения издержек, обеспечение роста прибыльности).

В качестве специфических внутренних факторов для предпринимательских структур выделены следующие факторы: размер предприятия и экстраполяция опыта взаимодействия в качестве физических лиц.

Размер предприятия связан с такими важными характеристиками, как наличие банковского счета и уровень цифровизации предприятия. Важнейшим инструментом взаимодействия кредитных организаций и предпринимательских структур является банковский счет, а в случае цифрового взаимодействия - банковский счет с дистанционным доступом.

Экстраполяция опыта взаимодействия в качестве физических лиц подразумевает что должностные лица предприятий, взаимодействуя с кредитными организациями как физические лица, переносят свой 
опыт на взаимодействие кредитных организаций и юридических лиц, оказывая влияние на упрощение взаимодействия, например, катализируя использование мобильных приложений.

Для кредитных организаций в качестве специфического фактора выделена «специализация кредитной организации (изменение традиционной модели банковской деятельности)». Подразумевается необходимость трансформации кредитной организации в зависимости от выбранного пути, среди которых можно выделить два основных направления - создание цифровых кредитных организаций (как самостоятельных, так и интегрирования цифровых проектов в традиционные кредитные организации) и превращение кредитных организаций в универсальную технологичную компанию (создание экосистемы, предполагающей расширение границ традиционной банковской деятельности) [7] [9].

\section{Обсуждение}

В статье предложена группировка внутренних и внешних факторов, обусловивших изменение экономических отношений кредитных организаций и предпринимательских структур в условиях цифровой экономики. Данная группировка опирается на существующую теоретическую базу, но учитывает особенности обоих участников экономических отношений, предпринимательских структур и кредитных организаций, а также специфику цифровой экономки. Перечень факторов не является исчерпывающим, перечислены, по мнению автора статьи, самые значимые из них. Факторы представлены в качестве многоуровневого списка для более глубокой детализации группировки.

\section{Заключение}

Группировка внутренних и внешних факторов, обусловивших изменение экономических отношений кредитных организаций и предпринимательских структур в условиях цифровой экономики может быть востребована при поиске решений для повышения цифровизации экономики Российской Федерации, необходимой для обеспечения роста ВВП. По данным национального исследовательского 
университета «Высшая школа экономики» к 2030 г. рост ВВП будет более чем на половину связан с цифровизацией $(1,47 \%$ из $2,75 \%$ ежегодного прироста ВВП), в первую очередь в результате повышения эффективности и конкурентоспособности всех секторов экономики. $[1$, с. 38] Менее амбициозный прогноз, но также, безусловно, доказывающий целесообразность цифровизации содержит исследование Мак-Кинзи [3 с.4]: потенциальный эффект цифровизации экономики для ВВП к 2025 году оценивается в 4,1-8,9 трлн. руб., что составит 19-34\% общего увеличения ВВП.

Для роста уровня цифровизации необходимо оценить состояние выделенных факторов, обусловивших изменение экономических отношений кредитных организаций и предпринимательских структур в условиях цифровой экономики, при необходимости принять меры для устранения препятствий, стоящих на пути цифровизации.

Повышению уровня цифровизации экономики безусловно способствуют прочные экономические отношения секторов экономики, в том числе финансового и реального. Поскольку цифровизация банковского сектора протекает более быстрыми темпами, чем предпринимательских структур, возможно построение инклюзивной модели, в которой банковский сектор взял бы на себя ведущую роль интеграции предпринимательских структур в цифровую среду и таким образом способствовал бы повышению цифровизации экономики.

Информация о конфликте интересов. Авторы заявляют об отсутствии конфликта интересов.

Информация о спонсорстве. Исследование не имело спонсорской поддержки.

\section{Список литературы}

1. Абдрахманова Г.И., Вишневский К.О., Гохберг Л.М. и др. Что такое цифровая экономика? Тренды, компетенции, измерение: докл. к XX Апр. между-нар. науч. конф., Москва, 9-12 апр. 2019 г., Национальный исследовательский университет «Высшая школа экономики». М.: Изд. дом Высшей школы экономики, 2019, 85 с. 
2. Александров А. В. Факторы обеспечения конкурентоспособности предпринимательских структур // УЭкС. 2011. № 29 (5). C. $110-123$.

3. Аптекман А., Калабин В., Клинцов В. и др. Цифровая Россия: новая реальность, июнь 2017, исследование Мак-Кинзи. https://www. mckinsey.com/ru/our-insights

4. Банки и банковское дело: учебник для бакалавров / под ред. В.А. Боровковой. М.: Издательство Юрайт, 2014. 623 с.

5. Волкова А.А., Плотников В.А., Рукинов М.В. Цифровая экономика: сущность явления, проблемы и риски формирования и развития// Управленческое консультирование. 2019 №4. С. 38-49. https://doi. org/10.22394/1726-1139-2019-4-38-49

6. Главацкий В.Б., Зике Р.В. Основные факторы развития российской банковской системы // Российское предпринимательство. 2014. Том 15. № 1. C. 64-69, https://creativeconomy.ru/lib/8466

7. Зверькова Т.Н. Банки, fintech, экосистемы: новые формы взаимодействия в финансовом посредничестве // Азимут научных исследований: экономика и управление, 2020. № 2, С. 159-163.

8. Зингер О.А., Ильясова А.В. Факторы, влияющие на устойчивое развитие промышленных предприятий // Современные проблемы науки и образования. 2015. № 1-1. URL: https://science-education.ru/ pdf/2015/1/701.pdf

9. Клейнер Г.Б., Рыбачук М.А., Карпинская В.А. Развитие экосистем в финансовом секторе России // Управленец, 2020. Т. 11, №4. С. 2-15. DOI: 10.29141/2218-5003-2020-11-4-1.

10. Колесов П.Ф. Основные факторы, влияющие на конкурентоспособность банков // Современные научные исследования и инновации. 2012. № 11 URL: http://web.snauka.ru/issues/2012/11/18351

11. Удаленные перспективы // Коммерсантъ. https://www.kommersant. $\mathrm{ru} / \mathrm{doc} / 4013343$

12. Котляров И.Д. Типовые бизнес-стратегии участников финансового рынка в условиях финансово-технической революции // ЭКО, 2019, T. 49, №2. C. 135-152. DOI: http://dx.doi.org/10.30680/ECO0131-76522019-2-135-152 
13. Лаукс Д., Маколей Д., Норонха Э., Уэйд М. Цифровой вихрь: как побеждать диджитал-инноваторов их же оружием. Москва: Эксмо, 2018. 352 c.

14. Плотников В.А. Цифровизация производства: теоретическая сущность и перспективы развития в российской экономике // Известия Санкт-Петербургского экономического университета, 2018, №4. С. 16-24. https://doi.org/10.24891/ea.16.12.2238

15. Устюжанина Е.В., Сигарев А.В., Шеин Р.А. Цифровая экономика как новая парадигма экономического развития // Экономический анализ: теория и практика. 2017. Т. 16, №12. С. 2238-2253.

16. Хунгуреева И.П., Шабыкова Н.Э., Унгаева И.Ю. Экономика предприятия: Учебное пособие. Улан-Удэ, Изд-во ВСГТУ, 2004. 240 с.

17. Ines Ghazouani Ben Ameur, Sonia Moussa Mhiri «Explanatory Factors of Bank Performance Evidence from Tunisia» // International Journal of Economics, Fi-nance and Management, Vol. 2, N. 1, March 2013, pp. 143152. http://citeseerx.ist.psu.edu/viewdoc/summary?doi=10.1.1.671.7188

18. Sujatha Trivikram, Influence of Business environment on the success of an organization, October 26, 2016, https://myventurepad.com/influence-business-environment-success-organisation

\section{References}

1. Abdrahmanova G.I., Vishnevskij K.O., Gohberg L.M. Chto takoe cifrovaya ekonomika? Trendy, kompetencii, izmerenie: dokl. $k$ XX Apr. mezhdunar. nauch. konf., [What is digital economy? Trends, references, estimation: report for XX april international scientific conference] Moscow, 9-12 apr. 2019, National Research University Higher School of Economics. M.: Izd. dom Vysshej shkoly ekonomiki, 2019, 85 p.

2. Aleksandrov A.V. Faktory obespecheniya konkurentosposobnosti predprinimatel'skikh struktur [Factors of ensuring the competitiveness of entrepreneurial structures]. UEkS. 2011. No. 29 (5), pp. 110-123.

3. Aptekman A., Kalabin V., Klincov V., Kuznecova E., Kulagin V., YAsenovec I., Cifrovaya Rossiya: novaya real 'nost', iyun'2017, issledovanie Mak-Kinzi [McKinsey report Digital Russia: new reality]. https://www. mckinsey.com/ru/our-insights 
4. Banki i bankovskoye delo: uchebnik dlya bakalavrov [Banks and banking: textbook for bachelors]. Moscow: Yurayt Publishing House, 2014. 623 p.

5. Volkova A.A., Plotnikov V.A., Rukinov M.V. Tsifrovaya ekonomika: sushchnost' yavleniya, problemy formirovaniya i razvitiya [Digital economy: entity of the phenomenon, problems and risks of formation and development]. Upravlencheskoe konsul'tirovanie [Management consulting], 2019. №4, pp. 38-49. https://doi.org/10.22394/1726-1139-2019-4-38-49

6. Glavatsky V.B., Zike R.V. Osnovnyye faktory razvitiya rossiyskoy bankovskoy sistemy [The main factors in the development of the Russian banking system]. Rossiyskoe predprinimatel'stvo [Russian Entrepreneurship]. 2014. Volume 15. No. 1, pp. 64-69. https://creativeconomy.ru/lib/8466

7. Zver'kova T.N. Banki, fintech, ekosistemy: novyye formy vzaimodeystviya $\mathrm{v}$ finansovom posrednichestve [Banks, fintech, ecosystems: new forms of interaction in financial intermediation]. Azimut nauchnykh issledovaniy: eko-nomika i upravlenie [Azimuth of scientific research: economics and management], 2020, pp. 159-163.

8. Zinger O.A., Ilyasova A.V. Faktory, vliyayushchiye na ustoychivoye razvitiye promyshlennykh predpriyatiy [Factors influencing the sustainable development of industrial enterprises]. Sovremennye problemy nauki $i$ obrazovaniya [Modern problems of science and education]. 2015. No. 1-1, https://science-education.ru/pdf/2015/1/701.pdf

9. Kleyner G.B., Rybachuk M.A., Karpinskaya V.A. Razvitiye ekosistem v finansovom sektore Rossii [Development of ecosystems in the financial sector of Russia]. Upravlenets [Manager], 2020. V. 11, №4, pp. 2-15. DOI: 10.29141/2218-5003-2020-11-4-1

10. Kolesov P.F. Osnovnyye faktory, vliyayushchiye na konkurentosposobnost' bankov [The main factors affecting the competitiveness of banks]. Sovremennye nauchnye issledovaniya i innovatsii [Modern research and innovation]. 2012. No. 11 http://web.snauka.ru/issues/2012/11/18351

11. Udalennyye perspektivy [Remote perspectives]. Kommersant. https:// www.kommersant.ru/doc/4013343

12. Kotlyarov I.D. Tipovyye biznes-strategii uchastnikov finansovogo rynka v usloviyakh finansovo-tekhnicheskoy revolyutsii [Typical business strategies of financial market participants in the context of the financial 
and technical revolution]. EKO, 2019, Vol. 49, №2, pp. 135-152. DOI: http://dx.doi.org/10.30680/ECO0131-7652-2019-2-135-152

13. Laux D., Macaulay D., Noronha E., Wade M. Tsifrovoy vikhr': kak pobezhdat'didzhital-innovatorov ikh zhe oruzhiyem [Digital vortex: how to defeat digital innovators with their own weapon]. Moscow: Eksmo, 2018. 352 p.

14. Plotnikov V.A. Tsifrovizatsiya proizvodstva: teoreticheskaya sushchnost' i perspektivy razvitiya $v$ rossiyskoy ekonomike [Digitalization of production: the theoretical essence and development prospects in the Russian economy]. Izvestiya Sankt-Peterburgskogo ekonomicheskogo universiteta [Bulletin of the St. Petersburg University of Economics], 2018, №4, pp. 16-24.

15. Ustyuzhanina Ye.V., Sigarev A.V., Shein R.A. Tsifrovaya ekonomika kak novaya paradigma ekonomicheskogo razvitiya [Digital economy as a new paradigm of economic development]. Ekonomicheskiy analiz: teoriya i praktika [Economic analysis: theory and practice]. 2017. V. 16, №12, pp. 2238-2253. https://doi.org/10.24891/ea.16.12.2238

16. Khungureyeva I.P., Shabykova N.E., Ungayeva I.Yu. Ekonomika predpriyatiya: Uchebnoye posobiye [Enterprise Economics: Textbook]. UlanUde VSGTU Publishing House, 2004. 240 p.

17. Ines Ghazouani Ben Ameur, Sonia Moussa Mhiri «Explanatory Factors of Bank Performance Evidence from Tunisia». International Journal of Economics, Finance and Management, Vol. 2, N. 1, March 2013, pp. $143-$ 152. http://citeseerx.ist.psu.edu/viewdoc/summary?doi=10.1.1.671.7188 18. Sujatha Trivikram, Influence of Business environment on the success of an organization, October 26, 2016, https://myventurepad.com/influence-business-environment-success-organisation

\section{ДАННЫЕ ОБ АВТОРЕ}

Генеральницкая Елена Игоревна

e_generalnitskaya@mail.ru

\section{DATA ABOUT THE AUTHOR Generalnitskaia Elena Igorevna \\ e_generalnitskaya@mail.ru ORCID: 0000-0001-7881-4213}

